In der Rubrik "Literatur kompakt" dieser Ausgabe referieren und kommentieren diese Experten für Sie Arbeiten aus der internationalen Fachliteratur.

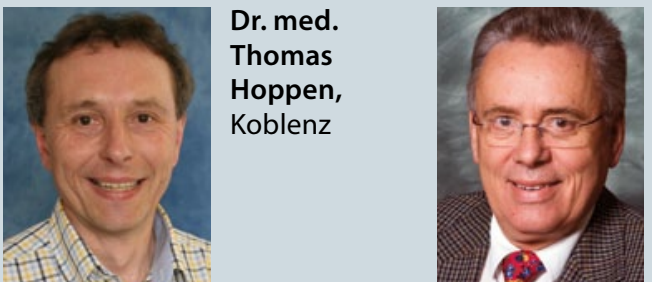

Dr. med. Hartmut Koch, Vechta

\section{Anwesenheit von Angehörigen bei der kardiopulmonalen Reanimation}

\section{Ob die Familienmitglieder eines Patienten bei der Reanimation anwesend sein sollten oder nicht, ist seit Jahren Gegenstand der Diskussion. Die mögliche Behinderung medizinischer Maßnahmen steht dem Wunsch des letzten Verabschiedens gegenüber. Wie verhält es sich mit der posttrauma- tischen Belastung bei den Angehörigen?}

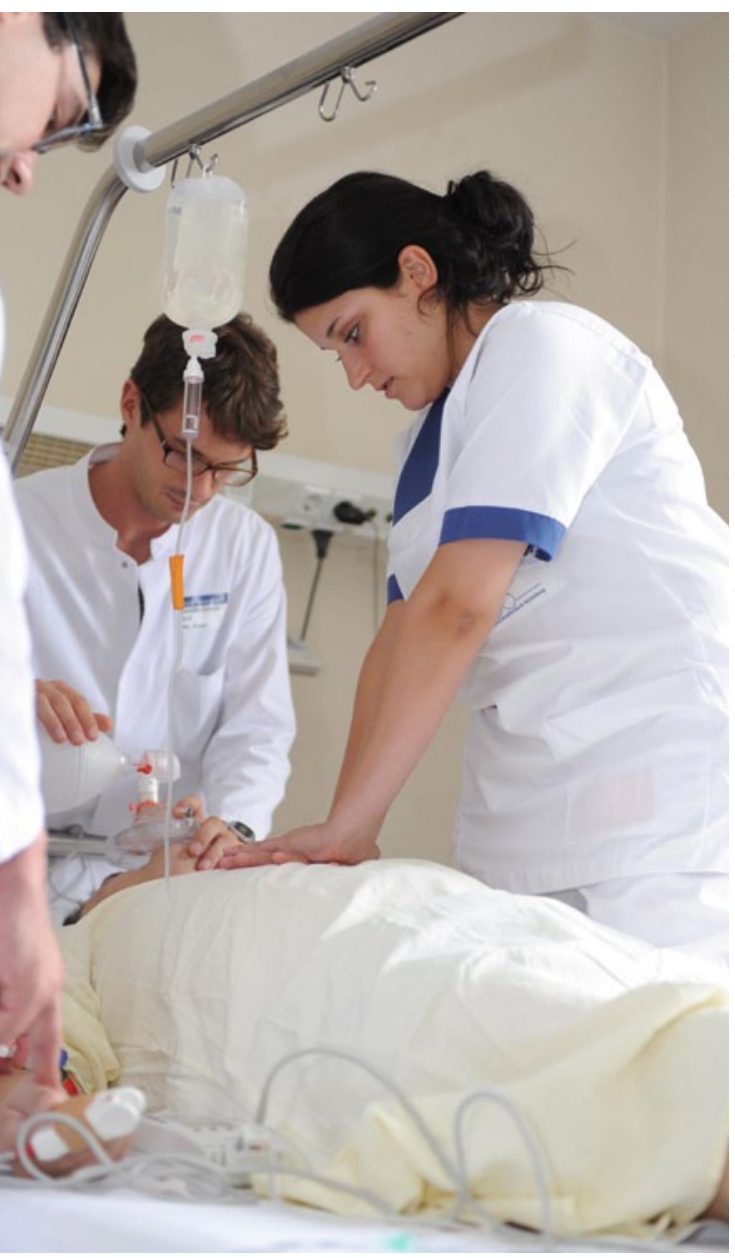

I Rahmen einer französischen Studie wurden 15 Notarztteams in eine Interventionsgruppe (acht Teams) und eine Kontrollgruppe (sieben Teams) randomisiert. 266 Familienangehörige der Interventionsgruppe nahmen auf Einladung an der Reanimation teil und wurden danach strukturiert betreut. In der Kontrollgruppe $(n=304)$ wurde eine Anwesenheit nur auf ausdrücklichen Wunsch der Angehörigen zugelassen. Symptome einer posttraumatischen Belastungsstörung (PTSD) bei den Angehörigen an Tag 90 war der primäre Endpunkt; sekundäre Endpunkte bestanden in Ängstlichkeit und Depressivität, Effekte der Familienpräsenz auf die medizinischen Leistungen, Befindlichkeit des Reanimationsteams und juristische Konsequenzen.

In der Interventionsgruppe waren $79 \%$, in der Kontrollgruppe $43 \%$ der Angehörigen bei der Reanimation zugegen. PTSD-Symptome traten in der Kontrollgruppe signifikant häufiger als in der Interventionsgruppe auf $(p=0,004)$. PTSD-Symptome fanden sich zudem

Die Angehörigen werden weniger traumatisiert, wenn sie bei der Wiederbelebung anwesend sein können. häufiger bei Angehörigen, die der Reanimation nicht beigewohnt hatten $(\mathrm{p}=$ 0,02 ). Zeugen der Wiederbelebungsmaßnahmen boten auch weniger Symptome von Ängstlichkeit und Depressivität. Weder der medizinische Ablauf noch der Erfolg der Reanimation wurden durch das Beisein der Angehörigen beeinflusst. Für das Reanimationsteam änderte sich das Stressniveau nicht. Juristische Konsequenzen fehlten ebenfalls.

Jabre $P$ et al. Family presence during cardiopulmonary resuscitation. $\mathbf{N}$ Engl J Med 2013; 368: 1008-18

Kommentar: Diese - von einer öffentlichen Institution finanziell unterstützte - randomisierte Studie belegt mit guten Argumenten eine in vielen Ländern mittlerweile anerkannte Praxis: die Anwesenheit naher Angehöriger bei der Reanimation. In den aktuellen ERC-Empfehlungen wird dieses Vorgehen positiv thematisiert. Unbedingt bedacht werden muss jedoch, dass es sich um eine Entscheidung handelt, die primär bei den Angehörigen liegt. Für die Anwesenheit von Angehörigen während einer außer- oder innerklinischen Reanimation sollten zudem einige Aspekte beachtet werden: Wahrung der Vertraulichkeit, verständnisvoller Umgang mit kulturellen und sozialen Unterschieden, Verwendung strukturierter Protokolle, Befolgung eines möglicherweise vorliegenden Patientenwunsches und Begleitung der Angehörigen durch speziell ausgebildete Teammitglieder. Auch wenn es sich bei dieser Studie ausschließlich um ältere erwachsene Patienten unter Reanimation handelte, haben die $\mathrm{Er}$ gebnisse nach eigener Einschätzung auch Bedeutung für die Kinderreanimation!

Dr. Thomas Hoppen 